\title{
LA INTERACCIÓN PEDAGÓGICA EN UN ENTORNO VIRTUAL DE APRENDIZAJE: UNA MIRADA ETNOGRÁFICA'
}

Tania Meneses Cabrera ${ }^{2}$ Patricia Ruiz Perdomo ${ }^{3}$

\section{Resumen}

El artículo expone de manera general una investigación concluida sobre el aprendizaje colaborativo dentro de un entorno virtual de aprendizaje y presenta con mayor relevancia el diseño metodológico de tipo cualitativo basado en la etnografía virtual, que se adelantó con estudiantes y directivos en la Universidad Nacional Abierta y a Distancia (UNAD) de Colombia. Se da prioridad a los aspectos metodológicos del trabajo, habida cuenta del auge de los estudios en cibercultura y la escasa reflexión que se hace sobre los diversos enfoques metodológicos para abordar un espacio social, antropológico y pedagógico emergente para las ciencias sociales. Los resultados se presentan a manera de hallazgos a partir del objetivo general de la investigación y en las conclusiones se aportan elementos relativos a la discusión sobre la fundamentación y el diseño metodológico, las técnicas e instrumentos de investigación aplicados a los entornos virtuales de aprendizaje.

\section{Palabras clave}

Aprendizaje colaborativo, Foros de discusión en línea, Cursos virtuales, Etnografía virtual.

\section{EDUCATIONAL INTERACTION IN A VIRTUAL LEARNING ENVIRONMENT: ETHNOGRAPHIC LOOK}

\section{Alistract}

The article presents a general view of an investigation culminated on collaborative learning within a virtual learning environment and emphasizes the qualitative methodological design based on virtual ethnography. The research was developed with students and executives at the Universidad Nacional Abierta y a Distancia (UNAD) of Colombia. Priority is given to the methodological issues of the research, taking into account the rise of cyberculture studies and little reflection given on methodological approaches to address a social, anthropological and pedagogical emerging in the social sciences. The results are reported as findings from the overall objective of the research and conclusions are provided elements for discussion on the rationale and study design, techniques and research tools applied to virtual learning environments.

\section{Keyword}

Collaborative learning, Online discussion forums, Virtual courses, Virtual ethnography

1 El artículo presenta información relativa a la metodología empleada en la investigación con apoyo Institucional, culminada y titulada: El Campus Virtual de la Universidad Nacional Abierta y a Distancia (UNAD) como espacio para el Aprendizaje Colaborativo, realizada por el Grupo Cibercultura y Territorio de la UNAD. Reporte de caso

2 Docente a la Universidad Nacional Abierta y a Distancia - UNAD. Nacionalidad Colombiana. Socióloga de la Universidad Nacional de Colombia y Magíster en Educación de la Universidad Santo Tomás en el 2009. tania.meneses@unad.edu.co

3 Docente Universidad Nacional Abierta y a Distancia - UNAD. Magíster en Desarrollo Educativo y Social, con estudios de Doctorado en Educación a Distancia y Tecnología Instruccional de NOVA Southeastern University. patricia.ruiz@unad.edu.co 


\section{Introducción y Objetivo de la Investigación}

El propósito del estudio fue aportar al mejoramiento en la interacción pedagógica entre los estudiantes de las aulas virtuales de la Universidad Nacional Abierta y a Distancia (UNAD), mediante la caracterización de la participación de los mismos en los foros colaborativos de los cursos virtuales y establecer si éstos se constituyen en dispositivo para la construcción de aprendizaje colaborativo.

La investigación empezó por definir el concepto de aprendizaje colaborativo en la literatura y en el modelo pedagógico de la UNAD, con el fin de apropiar el concepto para los entornos virtuales y definir las categorías relacionadas con dicho concepto, que orientarían el estudio. Luego, se diseñó una metodología basada en la etnografía virtual, para el abordaje de las categorías planteadas y la descripción del comportamiento de los estudiantes en los foros de discusión en línea.

\section{Aproximación Teórica}

En esta investigación se construyó una fundamentación epistemológica en la que se articula el enfoque teórico con el diseño metodológico, que permite abordar el objeto de estudio de manera coherente. Desde esta perspectiva, el enfoque teórico se orientó hacia la reflexión sobre los objetos tecnológicos y su básica instrumentalidad dentro de los procesos educativos y de manera general en la sociedad del conocimiento; así mismo, sobre el aporte a la construcción colaborativa de éste; sin embargo, es necesario resaltar que la simple instrumentalidad ya no es operante dentro del desarrollo, organización y producción de conocimiento, en tanto los objetos tecnológicos se convierten en artefactos que promueven nuevas formas de aprender y construir el mundo que habitamos.
Lo anterior se presenta como un reto frente a diversas variaciones en los procesos educativos, donde muchos docentes parecen conformarse únicamente con el acceso propio de sus estudiantes a las tecnologías, desconocen que no sólo se trata de acceder a ellas, sino que por el contrario, el objetivo es el de "construir, validar, crear conocimiento e interactuar con los otros. La vida se transforma a partir del uso de los medios; no podemos ser sólo consumidores, sino que debemos tratar de mejorar nuestras habilidades y competencias" para movernos en el mundo (Gros, 2008, p. 10).

Por lo tanto, es necesario concebir la tecnología como artefacto, como un puente en los diversos procesos de aprendizaje, en tanto es un dispositivo sociocognitivo de creación y apropiación sociocultural que forma parte de la vida de todos los seres humanos. En otros términos, la construcción de aprendizaje colaborativo debe entenderse como aquella situación interactiva que mediada o no por dispositivos digitales pretende aunar los esfuerzos de un grupo determinado hacia el objetivo que juntos puedan aprender y en el que se generan nuevos espacios o entornos que conducen a la construcción de nuevo conocimiento.

Para generar un proceso comprensivo en esta dirección se hace un acercamiento conceptual no sólo del concepto de aprendizaje colaborativo, sino también a las concepciones de dispositivos e interfaz para la generación de aprendizaje colaborativo en escenarios virtuales de aprendizaje, la interacción como elemento fundamental e indicador del proceso pedagógico y la mediación como condición pedagógica. Además fue necesario precisar también elementos propios del problema de investigación y el contexto institucional donde se desarrolló, en este caso hablamos de Moodle como plataforma tecnológica y ambiente digital de los cursos, la Universidad integra los 
elementos mencionados en una estructura de curso homogéneo a las diferentes disciplinas, en donde se desarrollan las actividades para cumplir las fases de aprendizaje, a través del estándar CORE, reflejo de un cúmulo de propuestas para articular el modelo pedagógico de la UNAD con los ambientes digitales para el aprendizaje y validado internacionalmente como eje central de las actividades de aula en los cursos académicos a través de la mediación digital y finalmente la fundamentación conceptual propia de la institución a través del análisis de su proyecto académico pedagógico.

Bajo estos presupuestos, la fundamentación epistemológica del diseño metodológico permitió plantear interrogantes propios en los estudios en cibercultura, frente al uso de enfoques, métodos, técnicas e instrumentos de investigación convencionales, habida cuenta que los fenómenos sociales en la era digital, desbordan las posibilidades de las metodologías existentes, como lo expresa Arriazu (2007). En consecuencia, el diseño metodológico escogido para el presente estudio daría cuenta de la finalidad comprensiva, el abordaje y el desarrollo de los objetivos propuestos, guarda coherencia con el problema de investigación y con la fundamentación teórica, relativos a la comprensión de algunos elementos de la educación como un fenómeno social presente en los entornos digitales.

Dado que se trata de una investigación educacional, por el contexto en el que se desarrolló, se pretendió aportar a la construcción de un conocimiento científico a partir de indagaciones propias sobre fenómenos educacionales comprendidos como sistemas abiertos, contradictorios y con componentes multideterminados (Pérez, García, Nocedo y García, 2001). En tanto el investigador forma parte del sistema social y educacional que estudia, lo aborda a partir de sus propios valores y convicciones; esta implicación subje- tiva del investigador, lo obliga a utilizar adecuadamente los principios metodológicos para validar de manera científica y confiable el conocimiento construido.

Es así que se toman como referentes los aportes y la fundamentación metodológica de la investigación social desarrollada por Babbie (2000), en particular sobre la definición de técnicas, aplicadas a la investigación social en el ciberespacio.

La investigación se desarrolló desde un enfoque comprensivo basada en la etnografía, para el análisis situacional, se tuvo en cuenta que esta permite trabajar con las relaciones propuestas en los objetivos, se entendió por etnografía una descripción densa a partir de la observación de una multiplicidad de estructuras conceptuales complejas, de las cuales algunas están superpuestas o enlazadas entre sí, estructuras que son al mismo tiempo extrañas, irregulares, no explícitas y a las cuales el etnógrafo debe ingeniarse de alguna manera, para captarlas primero y para explicarlas después (Martínez, 1994).

En síntesis la etnografía es una metodología central en el campo educativo, ya que permite validar el conocimiento que se construye a partir de las dinámicas sociales, es fundamentalmente un método con el cual se realiza una práctica descriptiva y comprensiva de la observación y descripción de grupos sociales. Para este estudio se trabajó con las reflexiones en torno a propuestas de etnografía virtual, que han realizado experiencias de investigación en cibercultura las cuales, a su vez, toman los principios de la etnografía convencional y la reconfiguran y tratan de interpretar las redes telemáticas como un espacio y además como un territorio (Arriazu, 2007).

Algunos científicos sociales como Levy (2004) y Haraway (1991), se ubican en un territorio epistemológicamente construido para la investigación, el ciberespacio desde la reflexión de lo que Arriazu (2007), considera que es un nuevo territorio $y$ 
acogido el término ciberespacio formulado por Gibson (1984), el novelista de ciencia ficción, en su obra Neuromante, para designar esta nueva forma de apropiación del espacio en las redes electrónicas. En este sentido se tienen en cuenta los trabajos realizados desde la investigación social on line - off line que desde sus necesidades particulares han reflexionado y avanzado en una propuesta metodológica pertinente para este tipo de investigación desde el enfoque cualitativo e interpretativo, teniendo gran experiencia de los estudios en comunicación y sociología en la red.

De acuerdo con Arriazu (2007), en la introducción a Cybersociety de Hine, se destaca la necesidad de crear nuevas formas de hacer investigación para poder dar cuenta de las formaciones sociales no-tradicionales que ocurren on line. Cada disciplina ha empleado sus propios métodos adaptándolos lo mejor posible a los entornos del ciberespacio. El análisis cuantitativo, por ejemplo, señalaba un camino para explorar los usos que se daban a Internet al contabilizar y correlacionar la ocurrencia de algunos de los mensajes allí publicados (Haraway, 1991).

Desde estas precisiones, la presente investigación se caracteriza por ser un estudio analítico situacional en relación con el alcance que se plantea y usa como método la etnografía, en cuanto al tiempo es transeccional contemporáneo, como lo explica Hurtado (2010), ya que pretende dar cuenta de una situación presente en forma particular, intenta a partir de una información analizar los elementos que intervienen en una situación determinada, a través de los datos, que en este caso fueron obtenidos en el trabajo de campo a partir de hechos que ocurren en el momento de la investigación, en el periodo comprendido entre en los años 2009 y 2010.
En una primera aproximación al evento de estudio, se realizó una exploración documental de la temática y del contexto institucional en que se realizó el estudio, a manera de antecedentes propios de este campo del conocimiento y con el fin de establecer una lectura general desde el problema de investigación, que permita de manera paralela profundizar en la fundamentación teórica y decidir las técnicas e instrumentos pertinentes para el diseño metodológico.

Desarrollo de la Investigación (Metodología, descriptores, muestra, instrumentos y demás)

\section{Muestra}

El universo poblacional son estudiantes, hombres y mujeres, de distintas edades y contextos regionales, inscritos en cursos virtuales de la UNAD. De manera aleatoria los participantes son organizados en grupos de cinco o seis estudiantes, quienes conocen el procedimiento de participación en los foros virtuales de aprendizaje. Cada foro cuenta con el acompañamiento de un Tutor, encargado de presentar las indicaciones para la participación en el foro, así como la forma de evaluar los procesos y resultados esperados del mismo.

La investigación realiza la colección de datos a partir de muestreos por juicio y conveniencia, no estadísticos, lo cual es una posibilidad cuando la investigación privilegia el análisis de datos cualitativos y no propone comprobaciones, sino comprensiones (Babbie, 2000). Se hizo una revisión general en torno a los cursos por trabajar en campus, actividad que se centró en la observación de las intervenciones de estudiantes y tutores para determinar si estas se convierten en interacciones que promueven el aprendizaje colaborativo. 
La selección de cursos obedeció a la condición de que estuviese en oferta para el período 2010-I y que fuesen representativos de distintos campos de formación. Así, se seleccionaron los siguientes cursos: 1) Intervención Psicosocial en el Contexto Educativo, 2) Diseño de procesos productivos, 3) Etnoeducación: Práctica Pedagógica I, 4) Lógica Matemática y 5) Política Agraria y ambiental.

\section{Categorías de análisis}

A partir de la revisión de literatura y el análisis de procedimientos metodológicos seleccionados, se establecieron los términos que se observan en la Tabla 1, como categorías de análisis de acuerdo con la elaboración discursiva realizada por los diferentes actores vinculados a la investigación, los cuales se presentan en la siguiente matriz categorial.

Tabla 1. Matriz categorial

\begin{tabular}{|l|l|l|l|}
\hline Categorías / Actores & Tutores & Directores de Curso & Estudiantes \\
\hline Campus Virtual & & & \\
\hline Aprendizaje Colaborativo & & & \\
\hline Core (Diseño Instruccional) & & & \\
\hline Foro & & & \\
\hline Interacción Pedagógica & & & \\
\hline Mediación & & & \\
\hline Enfoque pedagógico & & & \\
\hline
\end{tabular}

\section{Técnicas e instrumentos}

Para dar cuenta de los objetivos de la investigación se diseñaron distintos instrumentos, enmarcados en un ejercicio etnográfico para su consecuente análisis. Por ello, se utilizaron las siguientes técnicas e instrumentos de investigación.

\section{Observación no participante}

La técnica aplicada a este ejercicio es la observación no participante, para el seguimiento e interpretación de las interacciones comunicativas que establecen en el aula. En este caso la no participación es una estrategia en la etnografía y consiste en mantener una distancia prudente entre el grupo cultural que se va a describir y quien investiga. Ello supone una no exposición a la influencia de los pareceres y prácticas del grupo cultural de modo que el investigador pueda registrar de manera objetiva el acontecer de la vida del grupo. Se construyó una ficha de observación con las categorías explicadas con ante- rioridad y que indagaba por información puntal de las interacciones realizadas por el grupo.

\section{Instrumento clasificación de mensajes}

Dado que las participaciones de los estudiantes y del Tutor se registran en el foro del curso virtual, se diseñó un instrumento tipo lista de cotejo para el análisis y clasificación de cada intervención se tuvo en cuenta el tipo de mensaje y el tipo de interacción según su rol como estudiante o tutor y los niveles de participación que se generaba.

\section{Técnica entrevista}

Como respaldo al ejercicio etnográfico, se realizaron también entrevistas no estructuradas, tomando aportes de la cartografía social. Se propuso trabajar con los entrevistados un modelo de recuperación de la memoria institucional y de identidad frente al proceso de transformación de 
la Universidad que permitiera identificar en el tiempo el paso del enfoque de educación a distancia tradicional al de una cultura de la colaboración propia de la era digital, a través de un mapa conceptual y una línea del tiempo.

La entrevista es una técnica que permite la recolección de información de manera profunda e intencional a través de los relatos de personas que son interlocutores válidos en el tema de interés, por lo cual se consideró necesario realizar entrevistas a profesionales considerados como expertos dado que tienen o tuvieron a cargo la toma de decisiones relativas al proceso de transformación pedagógica de los cursos en la UNAD.

La finalidad de esta técnica fue la de identificar dentro del proceso de desarrollo pedagógico del campus virtual de la UNAD, las condiciones, orientación y contexto bajo los cuales se generó el aprendizaje colaborativo como estrategia didáctica de aprendizaje mediado. Se construyó una entrevista no estructurada que permitiera un ejercicio de memoria y constructo mental a través de la elaboración de un mapa conceptual y una línea de tiempo para recuperar momentos de decisión que afectaran el diseño de los cursos y el surgimiento y desarrollo de los foros colaborativos.

Las personas seleccionadas para las entrevistas, se consideran interlocutores válidos en calidad de expertos, por su formación académica, su experiencia profesional y por el acompañamiento que han realizado al proceso de transformación pedagógica y tecnológica de la UNAD. Los entrevistados tuvieron los siguientes roles y responsabilidades en relación con el objeto de la investigación: a) Diseño tecnológico y administrativo del LMS Moodle, b) Líder académico y tecnológico, vinculado al diseño del Core o diseño instruccional de los cursos virtuales, c) Líder académico para el diseño de la metodología del trabajo académico en la UNAD, d) Líder académico y directivo para el diseño de lineamientos pedagógicos.

\section{Técnica mapa conceptual}

Para el encuentro con los expertos arriba mencionados, se propuso la representación gráfica de conceptos clave para el objeto de estudio, mediante un mapa conceptual, por considerarlo como una estrategia de organización de la información propia de los procesos de aprendizaje significativo dentro del constructivismo al relacionar y jerarquizar los conceptos, para dar cuenta de su interpretación.

\section{Técnica línea de tiempo}

La línea de tiempo se pensó como una posibilidad de cualificar la información del mapa conceptual y aportar de manera gráfica información que identifique eventos significativos en el tiempo; pese a que plantea un enfoque de linealidad de la historia, es un ejercicio de referenciación importante para observar comportamiento de una situación en el tiempo y valorarla en la actualidad. Fue necesario discutir en el grupo la pertinencia del uso de estas técnicas dentro de la entrevista y se aprobó, habida cuenta que el nivel académico y perfil de los entrevistados permitía proponer la complejidad de este ejercicio y obtener buenos resultados

\section{Técnica grupo focal}

Además se aplicó un grupo focal con la intención de generar en el proceso de recolección de la información formas de participación y de interacción de los actores con la investigación y desde una perspectiva que otras técnicas no lo permiten, este grupo permite explorar percepciones, creencias y concepciones fundadas en los participantes del proceso sobre el que se investiga y genera mucha riqueza de información para la interpretación y análisis, y da pistas de relaciones y correlaciones importantes para la com- 
prensión del problema de investigación. Como lo veremos más adelante cuando se especifica cada técnica y se soporta con los instrumentos construidos de manera particular para esta investigación.

Las preguntas orientadoras del grupo focal fueron las siguientes:

1. ¿Qué ventajas y desventajas tienen los cursos de la UNAD desde su condición de virtual - digital para el aprendizaje colaborativo?

2. ¿Cómo cualificar el diseño de los espacios colaborativos de los cursos virtuales de la UNAD, para permitir el aprendizaje colaborativo?

3. ¿Cuál es el futuro deseable para el campus virtual de la UNAD?

4. ¿Existe coherencia entre el campus virtual, el CORE y el enfoque pedagógico de la UNAD?

\section{Síntesis de técnicas}

En la Tabla 2 se presenta una síntesis de la selección y aplicación de técnicas en relación con los objetivos de la investigación.

Tabla 2. Síntesis de técnicas por objetivos.

\begin{tabular}{|c|c|c|c|}
\hline Objetivos & Técnica & Población & Instrumento \\
\hline \multirow{2}{*}{$\begin{array}{l}\text { 1. Definir el concepto de aprendizaje colaborativo } \\
\text { con referencia al modelo pedagógico de la UNAD. }\end{array}$} & Reseña & Documentos & Fichas de registro \\
\hline & Conversatorio & Grupo de investigación & $\begin{array}{l}\text { Web conference, sky- } \\
\text { pe y actas de reunión }\end{array}$ \\
\hline \multirow{2}{*}{$\begin{array}{l}\text { 2. Categorizar los factores: sujetos, escenarios y } \\
\text { conceptos que en el campus virtual están planteados } \\
\text { como dispositivos para la generación de aprendizaje } \\
\text { colaborativo }\end{array}$} & Grupo focal & $\begin{array}{l}\text { Estudiantes y tutores de } 5 \\
\text { cursos }\end{array}$ & Protocolo grupo focal \\
\hline & Entrevista a expertos & $\begin{array}{l}\text { Seis funcionarios del nivel } \\
\text { directivo de la UNAD, que } \\
\text { participaron en el proceso } \\
\text { de diseño del campus virtual }\end{array}$ & Formato de entrevista \\
\hline $\begin{array}{l}\text { 3. Visibilizar los factores metodológicos, pedagógi- } \\
\text { cos, didácticos y operativos, que constituyen el foro } \\
\text { colaborativo del aula virtual }\end{array}$ & Observación & $\begin{array}{l}\text { Estudiantes y tutores de } \\
\text { cinco cursos }\end{array}$ & Ficha de observación \\
\hline
\end{tabular}

\section{Análisis de resultados}

Los resultados se presentan a continuación con base en las categorías de análisis encontradas durante el desarrollo del proyecto.

\section{Campus virtual}

Algunos de los conceptos encontrados en el proyecto se refieren a aspectos de tipo tecnológico, pues la oferta académica virtual exige la mayor calidad en cuanto a la infraestructura e inversión humana y económica. Sin contar con plataformas adecuadas para mantener toda la actividad de interacción entre los estudiantes, los tutores y los contenidos más las activi- dades sincrónicas y asincrónicas propias de la educación virtual, se pone en riesgo el proceso pedagógico.

Así mismo, la adecuada configuración tecnológica y la capacitación a los Tutores y Estudiantes en el manejo de las plataformas, son un factor relacionado con la ágil navegación por los contenidos y actividades de los cursos. Si una plataforma no es amigable en cuanto a la publicación de los contenidos de los cursos o de los mensajes de los Estudiantes, se hace difícil la atención tutorial y el seguimiento a los mismos. Los participantes en la investigación presumen que este asunto es de fácil solución si se cuenta con una planeación técnica y pedagógica adecua- 
da, por ejemplo, en cuanto a eventos de inducción a estudiantes que recién ingresan al uso de estas tecnologías.

En cuanto a los aspectos de tipo administrativo, un elemento sobresaliente es contar con las condiciones adecuadas para la dedicación de los Tutores según el número de estudiantes por curso académico. No contar con acompañamiento pedagógico oportuno y de calidad, puede ser un factor de deserción estudiantil.

\section{Aprendizaje colaborativo}

Los participantes demuestran contar con diferentes conceptos de aprendizaje colaborativo, por lo cual proponen acordar términos para exigirse mutuamente cumplimiento en los resultados del mismo por parte de los Estudiantes y las razones por las cuales esta estrategia pedagógica se considera importante en su formación como profesional.

Le dan alta importancia al acompañamiento que da el Tutor en el proceso, lo valoran como el $50 \%$ del éxito de esta actividad, creen que dicho acompañamiento debe ser permanente, pues una realimentación que se realice al final del mismo carece de sentido.

Con referencia a la mediación que se usa para el aprendizaje colaborativo, los participantes del proyecto no encuentran grandes diferencias en cuanto a las dificultades de realizar actividades en grupo. Por el contrario, establecen que la mediación no determina el éxito en el aprendizaje, sino el acompañamiento que tengan durante el curso para evitar frustraciones.

\section{Core o diseño instruccional}

Sobre el diseño instruccional de los cursos virtuales, los participantes del proyecto manifiestan la necesidad de contar con herramientas como el chat y el wiki para diversificar las actividades pedagógicas. La evaluación les genera conflicto debido a que deben cumplir simultáneamente con un alto número de exámenes finales y actividades de los cursos. Específicamente el número de trabajos colaborativos es alto y piensan que esto perjudica la calidad de su desarrollo, de acuerdo al tiempo del período académico y las dificultades de la interacción.

Una observación sobresaliente se refiere a que el diseño de los cursos desconoce los diferentes estilos de aprendizaje derivados de la diversidad en las características de la población, en cuanto a edad, ubicación geográfica, cultura y oficios, entre otros,

Los Tutores y Directores de Curso consideran un asunto importante contar con autonomía suficiente para organizar la agenda y las actividades de los cursos. También sugieren que las actividades de calificación automática que no cuentan con intervención del Tutor pueden permanecer disponibles durante todo el período académico y no limitar las fechas de acceso.

\section{Interacción}

La preocupación con la interacción se relaciona con la calidad y frecuencia de la participación. En general, los Tutores observan el cumplimiento de la tarea sin esfuerzo adicional, lo cual plantea un desafío para aumentar los niveles de lectura y de búsqueda de información especializada.

La participación en un foro debe empezar por leer los mensajes previamente publicados, reflexionar sobre ellos para aportar y debatir como parte del proceso que conduce a un resultado concreto, pero exige del estudiante análisis y compartir información con los otros. Es más que un intercambio de información, es el análisis que se hace de manera compartida a la misma. El recurso del tiempo y las competencias en el uso de 
la tecnología por parte de los estudiantes son elementos que facilitan la interacción.

Nuevamente se vincula a la reflexión el tema de la asignación razonable de estudiantes por Tutor para acompañar la forma de interactuar y el cumplimiento de los objetivos de formación.

\section{Mediación}

La selección de la mediación exige precisar qué tipo de herramientas y competencias, se requieren para tomar los cursos, así como la calidad y tipo de materiales que se utilizan, como elementos que aportan a los resultados esperados de la formación. En ese contexto, el rol del Tutor es prioritario en el trabajo de mediación y para ello debe ser formado como mediador, con las competencias adecuadas para el diseño de estrategias pedagógicas y el acompañamiento que exige este tipo de pedagogía mediada.

Esto aporta a la sensación de acompañamiento de los estudiantes y a la atención y respuesta oportuna a sus inquietudes.

\section{Enfoque pedagógico}

Se recomienda que tanto los Tutores como los Estudiantes que recién ingresan a los programas de educación a distancia se familiaricen con la nueva metodología para precisar el concepto que tienen y desempeñar su rol con calidad y mantenerlo así con el paso del tiempo. El aprender haciendo es un método recomendado desde el comienzo de la formación. Sobre la evaluación los participantes sugieren diversificar las estrategias de acuerdo con el tipo de curso.

\section{Conclusiones}

La investigación educativa y en particular la relacionada con el auge del uso y apropiación de tecnologías de información y comunicación, ha demostrado amplia preocupación por lo relacionado con el uso de herramientas, la integración de software y otras cuestiones más técnicas, pero en lo relacionado con la investigación propiamente pedagógica de los fenómenos sociales asociados con la educación, se visualiza un campo de construcción de conocimiento fecundo para los aportes de los educadores investigadores.

Considerar el ciberespacio como un espacio socio antropológico y las relaciones e interacciones simbólicas que allí se dan como propias de la cibercultura, es una propuesta epistemológica que necesita ser reflexionada y validada no sólo como enfoque teórico, sino que ésta, a su vez, debe pensar lo metodológico de manera coherente para su comprensión y análisis, en la medida en que se construyen nuevos códigos y lenguajes comunicativos y se reconstruyen formas de interpretación de los fenómenos.

El diseño metodológico utilizado en la investigación, permitió una caracterización comprensiva e integral de los foros y las posibilidades de estos en la generación de aprendizaje colaborativo, en la medida en que se tuvo en cuenta tanto los dispositivos tecno pedagógicos, las condiciones de los sujetos así como las realidades en la interacción y la mediación, a través del uso de técnicas de recolección de información a profundidad, es el caso de la observación no participante, las entrevistas y el grupo focal con diseños propios para la población objeto y las condiciones de la investigación.

El trabajo colaborativo tal y como se usa actualmente desde la experiencia observada, debe realizar una necesaria transición hacia la construcción de procesos de construcción de conocimiento colectivo, que permitan en primer lugar resignificar el concepto y sentido de la colaboración en escenarios digitales de aprendizaje, para luego potenciar la herramienta y los dispositivos pedagógicos. 
Si bien las teorías sobre las ventajas del aprendizaje colaborativo y las aplicaciones muestran resultados positivos, implementar esta propuesta en los ambientes de aprendizaje digital, requiere revisar las condiciones concretas y los perfiles de los sujetos que interactúan de acuerdo con el rol que cumplen.

La colaboración y la solicitud de actividades colectivas generan resistencia en los estudiantes, persiste el mito que reafirma el trabajo colectivo como algo que no funciona, aunque se reconocen sus beneficios, los resultados dependen en gran medida de la actitud hacia el trabajo colaborativo, la autorregulación y la calidad de la mediación por parte del tutor.

Persisten los problemas clásicos de los estudiantes en cuanto a los procesos de lecto-escritura y comprensión de instrucciones para realizar las actividades, la mayoría de los estudiantes tiene en esta institución su primera experiencia de enfrentarse a textos y material digital, al igual que la necesidad de gestionar información en Internet, es un punto muy importante para trabajar, ya que hace falta formación previa en este sentido

\section{Referencias}

ARRIAZU, R. (2007). ¿Nuevos medios o nuevas formas de indagación? Una propuesta metodológica para la investigación social on line a través del foro de discusión. Forum Qualitative Sozialforschung, Vol 8, No 3. Recuperado el 15 de abril de 2008 de: www. qualitative-research.net

BABBIE, E. (2000). Fundamentos de la Investigación social. Universidad Chapman. Thompson Editores.

COBO, R y Pardo, K. (2007). Planeta Web 2.0. Inteligencia colectiva o medios fast food. Grup de Recerca d'Interaccions Digitals, Universitat de Vic. Flacso, México.

GROS, B. (2008). Aprendizajes, conexiones y artefactos: La producción colaborativa del conocimiento. Barcelona, Gedisa.

HARAWAY, D. (1991). A Cyborg Manifesto: Science, Technology, and Socialist-Feminism in the Late Twentieth Century. En Simians, Cyborgs and Women: The Reinvention of Nature. New York.

HURTADO, J. (2000). Metodología de la Investigación Holística. Quirón. SYPAL. Caracas, Venezuela.

LEVY, P. (2004). Inteligencia Colectiva. Organización Panamericana de la Salud. Washington. Recuperado el 12 de marzo de 2008 de:http:// inteligenciacolectiva.bvsalud.org

MARTÍNEZ, M. (1994). Lalnvestigación cualitativa etnográfica en Educación. Segunda Edición. Trillas, México.
MENESES, T. (2009). La inteligencia colectiva en la educación digital. Trabajo de investigación evaluado como meritorio en la Maestría en Educación. Universidad Santo Tomás. Bogotá.

PÉREZ, G., García, G., Nocedo, I., García, M. (2001). Metodología de la investigación educacional. Pueblo y educación, La Habana. 